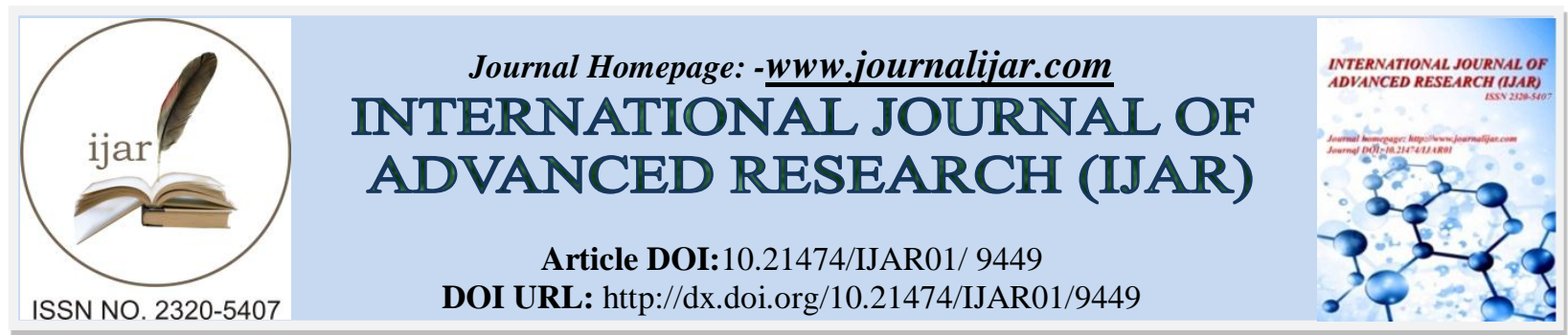

RESEARCH ARTICLE

\title{
QUANTUM SPACE IN THE CONTEXT OF PHILOSOPHY.
}

\begin{abstract}
J. Wang.
School of Physical science and Technology, Guangxi Normal University,Guilin, 541004, P. R. China.

\section{Manuscript Info}

Manuscript History

Received: 24 May 2019

Final Accepted: 26 June 2019

Published: July 2019

Abstract

The wave function, as the hardcore of quantum mechanics, consists in the quantum space. Consequently, the investigation for quantum space becomes an important part in both physics and philosophy, as well as the core problem in the philosophy of quantum mechanics. The speciality of quantum space is also the logical foundation of quantum mechanics and plays a determinant role. In this paper, we consider the quantum space as the primary entity and discuss its properties in the context of philosophy. Finally, we discuss the necessity of road to gravitation from the quantum space.
\end{abstract}

Copy Right, IJAR, 2019,. All rights reserved.

\section{Introduction:-}

The concept of "space" has been a topic to be actively explored since the ancient Greek era. According to Aristotle, the "space" must be defined on the basis of matter and its motion, and the existence of matter depends on space. Until 1600s, Isaac Newton proposed the mechanical theory and introduced the concept of "absolute space" independent of matter, i.e. the space is the container of the natural phenomena, which is independent. In 1900s, Albert Einstein indicated, in the special relativity, that the space expands and contracts with the velocity of observers. While in general relativity, he proposed that the expansion and contraction of the space reveal the gravity between the objects. Therefore, the space is not only the container of physical phenomena, but also has close relation to the gravity, which is subject to the gravity and thus will expand or contract. However, in the superstring theory, the space is not pre-existing and its dimension can transform from one to another, according to the coupling constant. Consequently, there should be more fundamental factors behind it. The space is just the manifestation of the fundamental factor, which is emergent.

According to the emergence, the structure of matter is hierarchical, and each hierarchy has new property, which is emergent property beyond the law of fundamental particles. Likewise, the quantum space is also a container, which overtops and dominates the matter in it. Even without any substance, the quantum space still has reality. However, because the fundamental entity in quantum mechanics is considered as high-dimensional field or cosmological particles, there are many ontological presuppositions in analyzing the quantum theory. In order to study the quantum space, we should not only discuss the contents in the space, but also the concepts of presupposition about the contents.

However, the quantum space depends both on the wave-particle duality of quantum objects consisting in it and on the potential requirement of referent theory and presupposition theory. All the theories or concepts have their realistic standpoint. The "reality" relates closely to the context, which has different implication in different context. If our interpretation for one concept is subject to some given philosophical tendency, based on the presupposed

Corresponding Author:-J. Wang.

Address:-School of Physical science and Technology, Guangxi Normal University,Guilin, 541004, P. 
conceptual system and structure, we have different description and knowledge about the quantum entity. Therefore, the quantum space, as a fundamental entity, displays relative uncertainty.

We resort to physical language and clarify our knowledge about the quantum space, which naturally get in the linguistic problems of significance, referent, and presupposition, when illustrating the formal system of quantum mechanics. In addition, the intentional action of observers is also involved. The way of correlation of the intentionality problem and physical language to the quantum space depends on the context of given conceptual system. For example, whether the quantum space is described by wave function or both wave function and particles depends on the intention of the interpreter. Consequently, the quantum space presents the intentional characteristic.

In this paper, we use the philosophical context and analyze the structure and dimensions of the quantum space. Finally, we demonstrate its relations to gravitation and its road to quantum gravity.

\section{Structure of Quantum Space:-}

Regardless of one-particle or multi-particles, the wave function itself, as the unity of entity and structure, reflects the potential reality with entity structure. The reason why the one-particle wave function has the fruitful and latent structures is that it can be expanded as different and complete sub-wave functions, i.e. the one-particle wave function is divisible and can be decomposed according to the complete basis. The multi-particle wave function can manifest as entangled quantum entities by the causal interactions, and the sub-states in the entangling state forms the entangled structure. Therefore, the complexity of quantum system causes the complex structures of the quantum space. Confined in the context of quantum mechanics, the limitation of structure of quantum space is also the limitation of complexity of quantum system itself. The wave function, describing the quantum world, particles, and the interactions, obeys the Schrödinger equation,

$$
-\frac{\hbar^{2}}{2 m} \frac{\partial^{2}}{\partial x^{2}} \psi(x, t)+V(x) \psi(x, t)=i \hbar \frac{\partial}{\partial t} \psi(x, t)
$$

which is the foundation of linguistic form of quantum mechanics.

Because of the wave-particle duality of the quantum objects and Bohr's principle of complementarity, the quantum space and classical space should be complementary. The classical concept can just describe part of the physical picture and different levels of objects. The characteristic of underdeterminism of quantum phenomena is described by classical concept, and the quantum state is the symbolic representation for the quantum phenomena consisting of complementary classical pictures. Both the 3-dimensional observed space and the quantum space are fundamental spaces, the equality becomes the reality in the context of decoherence [1].

Because the measured state of quantum objects depends on the consciousness of observers, the structure of quantum space actually depends on how to interpret it, which describes the characteristics of the quantum system. However, the interpreting subjects have no any freedom both to choose the state vector basis and to measure the corresponding objects. The Hamiltonian, describing the interactions in quantum space that must have corresponding one in the 3dimensional ordinary space, can be independent of the measuring environment and context. As a result, the structure of 3-dimensional space reflects the structure of quantum space [2].

The structure of quantum space relates closely to itself and the interactions between the embedded presupposition bodies. Different interactions between either the particles or particle and wave function result in different symmetries, which thus bring about the structures of quantum space. In this sense, the choice of structure of quantum space depends on the intentions of the subjects. Once choosing the given context to describe and interpret the quantum space, different structure is just the mathematical description for the corresponding physical reality. Therefore, they are the same in observations.

\section{Dimension of Quantum Space:-}

The dimension of quantum space, as part of the quantum space, has obviously semantic multidimensionality, i.e. 3dimension or $3 \mathrm{~N}$-dimension. According to the complementary principle, the quantum mechanics just can describe the results of experiments, instead of the real world. However, the experimental results just can present and describe the phenomena in the 3-dimensional ordinary space [3]. According to Erwin Schrödinger, the wave function evolves in the $3 \mathrm{~N}$-dimensional configuration space for a $\mathrm{N}$-particles system [4], which just represents the quantum state of the $\mathrm{N}$-particles system and gives us to the knowledge of the eigen-state of observables describing the system [5]. Therefore, the wave function is just the mathematical tool used to describe the quantum characteristics of particles 
on the configuration space. However, It subsequently claimed, according to the wave function substantialism, that the wave function is the wave field in $3 \mathrm{~N}$-dimensional configuration space, which locates in the $3 \mathrm{~N}$-dimensional space, instead of the 3-dimensional ordinary space [6]. We cannot observe the realistic level of the $3 \mathrm{~N}$-dimensional configuration space [7], and it is the intrinsic dynamics that leads us to the understanding for the fundamental properties of the quantum world, which subsequently determine the quantum space and its structure $[8,9]$.

It can be found that the different understanding for dimension of quantum space depends on how to understand the quantum theory. If we stand on the point of scientific experiment, the 3-dimensional ordinary space keeps the local causality and thus is appropriate and logical. If we treat the quantum space as a container, it is the secondary concept and the emergent result of some more fundamental objects. The dimension of quantum space with multidimensionality, which reflects the overall structure of the quantum space, as the trinity of "object-relationproperty", has complexity in the aspect of epistemology. The complexity results from four pairs of contradiction, i.e. the contradiction between certainty and uncertainty, the contradiction between entity and tool, the contradiction between locality and non-locality, and the contradiction between logic and intuition.

It is the unobservability of the structure of quantum space that leads to the contextual dependence for the interpretation of dimension of quantum space. For all kinds of the interpretations, we should understand and analyze them according to given boundary condition and presuppose the boundary condition, i.e. correlated context. Different interpretations for quantum space and the structural relation determine the different semantic borders. In order to avoid the complexity, we should remove the complexity and multidimensionality on the level of epistemology.

\section{Road to Quantum Gravity:-}

When we discuss the structure of quantum space, it naturally involves the geometry of space-time and thus the effects of gravitation, which is described by Einstein's general relativity. Whether we consider the wave function as particles or fields, it carries energy and momentum, which will cause the space-time bending. While the curved space-time, in turn, has influence on the motion of the particles and fields in it, and then the evolution of wave function. Consequently, we cannot neglect the interaction between the structures of quantum space and their gravitation and should seek for the unification between quantum theory and gravitation.

In order to unify the standard quantum mechanics and gravitation, i.e. general relativity, we need to apply the canonical quantization, i.e. Hamiltonian form, to general relativity and then quantize the gravitation by usual method, which creates the canonical quantum gravity. In quantum gravity, the wave function describes the 3-dimensional geometry on the 3-dimensional Riemann configuration space, instead of the physical state. In the standard quantum mechanics, the interactions between particles are local interactions, and the superposition of different states of each quantum object can independently represent the superposition state. However, the interactions between the structures of quantum space realize by the gravitational quanta, which is long rang. Accordingly, the quantum space is a non-local concept and physical entity. Owing to the principle of complementarity, we cannot determine some dynamical variable and its variance rate with respect to time. As a result, the uncertainty principle doesn't allow us to give the determinism of evolution of space with respect to time to any physical significance, in the framework of canonical quantum gravity. The time related Schrödinger equation is not appropriate in such framework, because the state vector is buried in the Hamiltonian. The Hamiltonian, describing the interactions of the structures, doesn't evolve with time. Consequently, the formed quantum theory is independent of the background and becomes the theory of default time, which has significant influence on the development of space-time realism.

\section{References:-}

1. G. Bacciagaluppi, "The Role of Decoherence in Quantum Theory", The Stanford Encyclopedia of Philosophy, 2008

2. M. Schlosshauer, "Decoherence and the Quantum-to-Classical Transition", Berlin: Springer, 2007

3. A. Ney, "The Status of Our Ordinary Three Dimensions in A Quantum Universe", Wiley Periodicals, 46,534 (2012)

4. B. Monton, "Quantum Mechanics and 3N-dimensional Space", Philosophy of Science, 2006

5. B. Monton, "Against 3N- Dimensional Space", D. Albert, A. Ney, in The wave Function: Essays in the Metaphysics of Quantum Mechanics, Oxford University Press, 2013

6. M. Bohn, "Physics in My Generation", Pergamon Press, 1956

7. J. North, "The 'Structure' of Physics: A Case Study", Journal of Philosophy, 106, 58 (2009) 
8. J. S. Bell, "Speakable and Unspeakable in Quantum Mechanics", Cambridge University Press, 1987

9. P. Lewis, "Life in Configuration Space", British Journal for The Philosophy of Science, 55, 714 (2004). 